disinfecting chemicals in the workplace $(\mathrm{p}=0.01 ; \mathrm{OR}=$ $17.306)$ and the absence of occupational reclassification $(\mathrm{p}=$ $0.01 ;$ OR $=21.567)$.

Conclusion This study has revealed skin-related QoL is significantly more impaired in women. Several studies have reported a similar result. This could be explained by the fact that traditionally, in Tunisia, women have been predominantly responsible for household chores, and therefore more exposed to substances that can trigger dermatological conditions. In addition, the aesthetic consequences of skin pathology do not have the same impact on men and women.

\section{P-363 SEX DIFFERENCES IN MANAGING ACCIDENTAL BLOOD EXPOSURE IN A TUNISIAN PUBLIC HOSPITAL}

'Amira Omrane, Harrathi Chayma, Meriem Ben Hassine, Lamia Bouzgarrou, Aouatef Mahfoudh, Taoufik Khalfallah. ${ }^{1}$ Faculty of Medicine of Monastir, Tunisia

\subsection{6/OEM-2021-EPI.290}

Accidental blood exposure (ABE) is major problem of occupational safety among health care workers. This work aimed to assess the gender differences among health care workers of a public hospital in the central region of Tunisia.

Methods An exhaustive retrospective study was conducted over a period of fourteen years, concerning $\mathrm{ABE}$ reported in the Department of Occupational Medicine of a public hospital in the central region of Tunisia (Mahdia). The data collected were related to socio-professional characteristics of victims, circumstances of the accident and immediate and follow-up care. Gender groups were compared.

Results A total of 650 ABE were reported during the study period. Victims were predominately female (sex ratio $=0.47$ ), mean aged $32.1 \pm 9.4$ years and having a mean job tenure of $5.5 \pm 7.7$ years. Paramedics represented 28.3\%. AEB were caused by a needle stick in $82.6 \%$ of cases. Needle recapping was the direct cause in $10.6 \%$ of cases. Only $47.1 \%$ of caregivers were wearing gloves at the time of $\mathrm{ABE}$. Women were more exposed to $\mathrm{ABE}$ with a higher risk of contamination, but with no statistically significant difference $(p=0.33)$. Immediate care was in accordance with universal recommendations in $34.1 \%$ of cases and less adequate among women $(p=0.05)$. The initial serology was prescribed in $82.6 \%$ of cases, that of the $3 \mathrm{rd}$ month in $5 \%$ of cases and that of the 6 th month in $1.2 \%$ of cases. Women were less adherent to follow-up with a statistically significant difference at six months $(p=0.016)$. Hepatitis B vaccination schedule was correctly followed in $79 \%$ of cases. Women were more adherent than men $(p=0.029)$. Logistic regression analysis did not reveal gender as a predictor of vaccination status $(\mathrm{p}=0.211)$.

Conclusion The current study has revealed a high prevalence of $\mathrm{ABE}$ mainly among female health care workers with unsatisfactory management, including inadequate immediate care and incomplete post-accident follow-up.

\section{P-364 INTERACTIONS AMONG SHIFT WORK DOMAINS AND EMPTY CALORIE FOOD/BEVERAGE CONSUMPTION}

${ }^{1}$ Ting-Ti Lin, Shannon Zenk, Pamela Martyn-Nemeth, Lisa Tussing-Humphreys, Kathleen M Rospenda, Chang Park, Mary C Kapella. ${ }^{1}$ National Defense Medical Center, Taiwan

10.1136/OEM-2021-EPI.291
Introduction Shift work has been linked to increased consumption of empty calorie food/beverages. However, the majority of studies investigating associations between shift work and empty calorie food/beverage consumption has been focusing on the impacts of shift timing. Little is known about how other domains of shift work contribute to empty calorie food/ beverage consumption.

Objectives The purpose of this study was to examine associations between shift work and empty calorie food/beverage consumption, focusing on other domains of shift work and their interactions.

Methods This was a 14-day intensive longitudinal study with ecological momentary assessment. A convenience sample of 80 Taiwanese hospital registered nurses were recruited. During the study period, employing a 21-item food checklist, participants were prompted four times daily to report their empty calorie food/beverage consumption on a smartphone. Three domains of shift work: shift timing, intensity, and speed were derived from the registry-based work schedules. Three-level mixed-effects regression models were used to test hypotheses.

Results A total of 2,444 momentary observations from 77 participants were included in the final analysis. Findings suggested that high night shift intensity was associated with an increased likelihood of sugar-sweetened beverage intake (AOR $=1.64$, 95\% CI [1.01, 2.68]). Shift speed moderated associations between sugar-sweetened beverage consumption and work shift intensity or shift timing. However, associations between night shift intensity and empty calorie food/beverage consumption did not vary by shift speed.

Conclusions This study suggested how shift assignments might contribute to workers' empty calorie food/beverage consumption. Therefore, it would be beneficial to rotating shift workers' eating behaviors and overall health if the identified hazardous shift schedule can be avoided.

\section{P-365 PROMOTING SENTINEL SURVEILLANCE PROGRAMS FOR ANTIMICROBIAL RESISTANCE IN CANADIAN SALMON AQUACULTURE, A POSSIBLE AND UNDERSTATED OCCUPATIONAL HEALTH HAZARD}

${ }^{1}$ Cory Ochs, Barb Neis, Kapil Tahlan, Atanu Sarkar. 'Memorial University of Newfoundland and Labrador, Canada

\subsection{6/OEM-2021-EPI.292}

Introduction Canadian salmon aquaculture is a high-risk industry with injury rates that surpass provincial averages in Atlantic Canada, yet few publications address occupational health hazards. Antimicrobial resistance (AMR) emergence is a growing public health concern, and the marine aquatic environment with its rich microbiota is particularly vulnerable to selection of AMR. Antibiotic use in the industry and other anthropogenic activities that result in the deposit of pollutants contaminated with antibiotics into the marine environment can together amplify selective pressure propagating AMR. Similar to terrestrial animal production facilities, there is concern for the development of hotspots for occupational exposures to AMR among aquaculture workers. As the fastest growing food production network globally, the aquaculture industry has been appealed by the Food and Agriculture Organization, among others, to standardize monitoring and to generate an evidence base to better understand the aetiology of AMR emergence in aquaculture settings. 
Objectives This case study will identify Canadian policies and practices that promote antimicrobial stewardship and surveillance in the aquaculture industry.

Methods i) Compare antibiotic use by Canadian salmon aquaculture to other global industry leaders. ii) Compare regulatory regimes and surveillance strategies across industry leaders.

Results Prescribed antibiotic use in Canadian salmon aquaculture exceeds that of Norway, the industry leader, which has implemented an array of strategies to drastically reduce antibiotic use since the 1990s. Unlike Norway, Canadian aquaculture lacks monitoring programmes for AMR and, furthermore, has yet to document possible occupational exposure pathways to this hazard. Current data repositories do not elucidate health risks associated with AMR emergence in aquaculture settings.

Conclusion Canadian salmon aquaculture has an opportunity to lead the country's animal production industries in the development of a standardized sentinel surveillance network to accommodate formal risk analyses and early warning systems. Continuous AMR surveillance coordinated with current public health monitoring would promote health protective strategy development and antimicrobial stewardship within the country.

\section{P-368 OCCUPATIONAL BIOLOGICAL LIMIT DERIVATION PROCESS AND BIOLOGICAL LIMIT VALUES FOR SEVERAL PRIORITY SUBSTANCES}

${ }^{1}$ Nolwenn Noisel, Farida Lamkarkach, Claude Viau, Fatoumata Sissoko, Christophe Rousselle, Domnique Brunet. 'University of Montreal, Canada

\subsection{6/OEM-2021-EPI.293}

Introduction Biomonitoring and atmospheric metrology are complementary approaches to assess occupational exposure to chemicals. The ANSES working group on biomarkers of exposure (WGBME) has developed an approach to derive Biological Limit Value (BLV) for occupational priority substances.

Objectives The aim of this commuication is to present the approach as we as the derived BLV for the priotity substances.

Methods Based on available data and using a decision tree, 4 types of BLV may be derived: a BLV based on a health effect for substances with threshold effects, a BLV based on an Occupational Exposure Limit (OEL), a BLV based on a cancer risk level $(10-4,10-5$ or $10-6)$ or a theoretical value called 'pragmatic BLV'. When knowledge on the relationship biomarker-health effects or biomarker-exposure is lacking, no BLV is derived. Whenever possible, a Biological Reference Value (BRV) based on the 95th percentile of a non-occupationally exposed population is also proposed. BRVs are not risk-based but are part of the preventer's toolbox.

Results Since 2011, 16 substances were assessed by the ANSES WGBME. Detailed information has been published in scientific reports which are publicly available on the ANSES website. Lead and Cadmium were the only chemicals for which BLVs based on relationship between health effect and biological levels were derived: lead BLV of $180 \mu \mathrm{g}$.L-1 based on neurological effects and urinary cadmium (5 $\mu \mathrm{g} . \mathrm{g}-1$ creatinine) and blood cadmium (4 $\mu$ g.L-1) based on nephrotoxicity. BLVs (urinary concentrations) based on OELs were derived for cobalt (5 $\mu$ g.g-1 creatinine), dichloromethane (0.2 mg.L-1) and styrene $(40 \mu$ g.L-1). A pragmatic BLV based on OEL was calculated for chromium VI $(2.5 \mu \mathrm{g} . \mathrm{L}-1)$. No BLV was based on cancer risk level. In addition, no BLVs but BRVs were proposed for substances such as acrylamide, beryllium, butadiene and some phtalates.

Conclusion This expertise from the ANSES WGBME has led to derive several BLV to prevent health effects in workers or to control exposure to contaminants. BLV and BRV help occupational physician to unfold prevention program and surveillance in occupational settings.

\section{P-372 SAFETY DATA SHEETS AS A HAZARD COMMUNICATION TOOL: AN ASSESSMENT OF SUITABILITY AND READABILITY}

${ }^{1}$ Kevin Ho, Thomas Tenkate. ${ }^{1}$ Ryerson University, Canada

\subsection{6/OEM-2021-EPI.294}

Introduction Safety data sheets (SDSs) are printed materials designed to communicate the hazards associated with using chemicals/hazardous products in the workplace. SDSs are highly technical in nature, often containing dense, ambiguous text, which places considerable comprehension demands on workers, especially those with lower literacy skills.

Objective To assess the suitability and readability of SDSs as a hazard communication tool for workers.

Methods A random sample of 50 SDSs compliant with WHMIS 2015 were extracted from the CCOHS m(SDS) database. The Suitability of Materials (SAM) tool, originally designed to evaluate patient education materials, was used to assess SDSs for content, literacy demand, use of graphics, and layout/typography. To account for legislated content requirements under WHMIS 2015, an amended SAM tool was also developed for scoring. Readability software was used to determine the reading grade level required to understand SDSs based on several common formulas/indices.

Results When the original SAM tool was used, the mean total SAM score was 'not suitable' (30.22\%). When the amended SAM tool was used, the mean total SAM score increased to 'adequate' (64.43\%). The mean readability scores were as follows: Flesch-Kincaid Grade Level (10.2), Gunning-Fox Index (8.5), Coleman-Liau Index (12.2), and Simple Measure of Gobbledygook index (10.2).

Conclusion Even though the amended SAM tool was better able to identify content-related issues specific to SDSs, the use of SDSs as a hazard communication tool needs improvement. The SDSs analyzed required a reading grade level between the 8 th and 12th grades. These levels exceed the 6th-grade reading level recommended to ensure that $75 \%$ of adult Americans can read the material without difficulty. Overall, chemical/hazardous product manufacturers should use readability assessments together with the amended SAM tool when writing SDSs to ensure that the written information is easily understandable for all audiences.

\section{P-373 IMPACT OF MUSCULOSKELETAL DISEASES ON HEALTH- RELATED QUALITY OF LIFE AMONG OFFICE FEMALE WORKERS.}

${ }^{1}$ Amira Omrane, Meriem Zerai, Harrathi Chayma, Aouatef Mahfoudh, Taoufik Khalfallah. ${ }^{1}$ Faculty of Medicine of Monastir, Tunisia

10.1136/OEM-2021-EPI.295 